\title{
Lipid absorption and overall intestinal lymphatic transport are impaired following partial small bowel resection in mice
}

\author{
Emily J. Onufer
}

Washington University in St. Louis

Rafael S. Czepielewski

Washington University in St. Louis

\section{Yong-Hyun Han}

Kangwon National University

Cathleen M. Courtney

Washington University in St. Louis

\section{Stephanie Sutton}

St. Louis Children's Hospital

\section{Anne Sescleifer}

St. Louis University

\section{Gwendalyn J. Randolph}

Washington University in St. Louis

Brad W. Warner ( $\boldsymbol{\sigma}$ brad.warner@wustl.edu )

St. Louis Children's Hospital

\section{Research Article}

Keywords: short bowel syndrome, small bowel resection, lymphatic remodeling, intestinal failureassociated liver disease

Posted Date: November 29th, 2021

DOI: https://doi.org/10.21203/rs.3.rs-1079731/v1

License: (c) (i) This work is licensed under a Creative Commons Attribution 4.0 International License. Read Full License 


\section{Abstract}

Short bowel syndrome (SBS) is associated with diminished levels of serum fats caused by unknown mechanisms. We have shown that mesenteric lymphatics remodel to a more primitive state one week after small bowel resection (SBR); therefore, this study focuses on the effect of chronic lymphatic remodeling and magnitude of resection on intestinal fatty acid uptake and transport. C57BL6 and Prox1 creER-Rosa26 ${ }^{\mathrm{LSL}}$ TdTomato (lymphatic reporter) mice underwent $50 \%$ or $75 \%$ proximal SBR or sham operations. Functional transport of lipids and fecal fat content was measured and lymphatic vasculature was compared via imaging. There was a significant reduction in functional transport of cholesterol and triglyceride after SBR with increasing loss of bowel, mirrored by a progressive increase in fecal fat content. We also describe significant morphological changes in the lymphatic vasculature in both the lamina propria and mesentery. Intestinal lymphatic drainage assay in vivo demonstrated a marked reduction of systemic absorption after resection. Intestinal lymphatic vessels significantly remodel in the setting of chronic SBS. This remodeling results in impaired intestinal transport of fat via the compromised lymphatic architecture, contributing to decreased fatty acid uptake. We believe that these changes may contribute to the development of IFALD, a major morbidity in patients with SBS.

\section{Introduction}

Short bowel syndrome (SBS) is a morbid clinical condition resulting from the massive loss of small intestine due to surgical resection. While such resections may be clinically necessary in settings as diverse as necrotizing enterocolitis, trauma, or inflammatory bowel disease, these radical surgeries have their own potentially adverse consequences. The remaining bowel may be unable to absorb and/or digest substantive nutrients for maintenance and growth(1-3). This failure can arise despite the remnant bowel undergoing a compensatory adaptation response to increase function via expansion of intestinal surface area. Consequently, many SBS patients still require parenteral nutrition for additional supplementation(4).

One class of these important macronutrient in the context of SBS is dietary fat. While fatty acids and cholesterol can be synthesized de novo, the uptake of these lipids occurs concomitant with essential fatty acids and lipophilic vitamins that are not synthesized and must be acquired from the diet. Lipid absorption relies on the generation of lipoproteins called chylomicrons that are assembled and secreted by intestinal epithelial cells in the proximal small bowel and subsequently transported into intestinedraining lymphatic vessels(5). Bile acids, derived from hepatic cholesterol metabolism, reach the proximal gut through the bile duct, where they facilitate emulsification of dietary fats that allows epithelial uptake and repackaging of ingested lipids into chylomicrons. In turn, re-absorption of biliary bile acids occurs in the distal small intestine(6). Diet-derived plant sterols, such as campesterol, enter the body via chylomicrons and thereby serve as a surrogate marker for dietary lipid absorption(7-10). When lipid absorption is impaired, as in patients with ileal dysfunction, the ratio of plasma lathesterol (a surrogate for endogenous cholesterol synthesis) to campesterol is frequently increased (11-13). Indeed, there is a significant reduction in campesterol in the plasma of SBS patients after withdrawal from parenteral nutrition(14), raising the possibility that these patients may fail to absorb lipid nutrients from 
the diet normally. While diminished intestinal epithelial cell populations is thought to account for malabsorption of lipids observed in patients with SBS, other possible contributing factors like failed transport of cargo through the lymphatic vasculature has not been assessed. Addressing these possibilities is critical to optimizing and improving clinical management of SBS patients.

We had previously reported extensive structural remodeling of mesenteric lymphatic vessels draining the intestine within a week after experimental small bowel resection (SBR) in mice(15). However, it remained unclear whether this remodeling persisted beyond an initial recovery from surgery and whether lymphatic transport was functionally impacted by the remodeling. Here, we reveal that lymphatic cargo, including but not limited to dietary lipids, fail to traffic from the gut to the host circulation or draining lymph nodes. These findings highlight the need to consider diminished lymphatic transport in therapeutic management of SBS.

\section{Results}

\section{Malabsorption of cholesterol and triglycerides after SBR}

At an early time point after resection, postoperative day 7, the distal intestine reprograms to a more proximal identity with an upregulation of genes associated with lipid handling and metabolism(16). In agreement with this reprogramming, we observed a progressive increase in expression of $A p o B$ in the proximal intestine and MTTP in the remaining distal intestinal segment, which regulate the assembly of chylomicrons, in the resected mice (Figure 1A-B; ANOVA $p<0.05$ and $p<0.01$, respectively). As we have previously shown in single-cell analysis at early time points after resection, there was an increase in the intestinal lipid-sensing gene FABP2 in the distal intestine (Figure 1B; ANOVA $p<0.005)(16)$.

Simultaneously, in the distal intestine, there was a decrease in the long-chain fatty acid transporter gene, $C D 36$, the cholesterol transporter gene, $A b c a 1$, and the bile acid binding gene, $F A B P 6$, expression in the distal intestine with increasing loss of bowel (Figure 1B; ANOVA $p<0.005, p<0.005$, and $p<0.05$, respectively). These data show that the intestine reprograms to promote lipid absorption and chylomicron assembly, leading us to test whether fat absorption normalized in concert with this reprogramming.

Despite the favorable programming of genes to orient toward fat absorption, with increasing loss of bowel, total serum cholesterol decreased with $75 \%$ resected mice having a $67 \%$ decrease compared to non-operated controls (Figure 2A; ANOVA p 0.0001 ). Serum campesterol, a marker of dietary cholesterol absorption, followed this same trend of decreased concentrations with increased extent of bowel resection (Figure 2B; ANOVA $p<0.001$ ). Lathosterol, an intermediate in cholesterol synthesis, also decreased in the serum as proportion of intestine resected increased (Figure 2C; ANOVA $p<0.05$ ). However, as the lathosterol to campesterol ratio increased with the magnitude of resection (Figure 2D), the extent of malabsorption appears greater than the decrease in cholesterol synthesis. To directly assess absorption of exogenous cholesterol, we performed a time-course following oral delivery of the fluorescently labeled cholesterol mimetic TOPFLUOR cholesterol. With increasing loss of bowel, there was a decreasing amount of TOPFLUOR cholesterol absorbed (Figure 2E-F; ANOVA $p<0.0001$ ). 
These data pointed to failed absorption of fat, leading to seek other ways to confirm this observation. Failed absorption of dietary fat would be expected to result from increased fat content in feces. At 1.5 years after SBR, with increasing loss of bowel after resection, fecal fat concomitantly increased (Figure $3 A$; ANOVA $p<0.0005$ ), a feature that held true when the data were normalized to fat intake (Figure 3B; ANOVA $p<0.005)$. To assess chylomicron transport via lymphatics, we performed a functional assay of lipid absorption using C16-Bodipy-labeled olive oil. This showed a decrease in chylomicron absorption with a lipid load over time in the resected mice (Figure 3C-D; ANOVA $p<0.0001$ ). Serum free fatty acids at 12-15 weeks after resection also followed this same trend (Figure $3 \mathrm{E}$; ANOVA $p=0.0007$ ).

These data indicate that failed intestinal lipid absorption is present in mice long after surgical resection. Overall, we conclude that fat malabsorption exists as a chronic consequence of SBR. This malabsorption exists despite gene expression in the intestine that should favor absorption. On the other hand, SBR promoted a $51 \%$ reduction in serum bile acids after an oral fat load, which may result in reduced secretion of bile acids in the intestinal lumen to promote lipid uptake (Figure $4 A-B ; p<0.05$ ). There may also be a role for lymphatic remodeling. That is, if lymphatic transport was impaired, malabsorption might occur even in the face of genes oriented to take up and package fats for transport. Consequently, we turned our attention to examining the status of the lymphatic vasculature.

\section{Intestinal mucosal lymphatic capillaries change after chronic SBR}

We have previously shown dilation of the upstream mucosal lymphatic capillaries in the remnant distal bowel seven days after resection(15), but it was unclear if over time the remodeled lymphatics returned to their baseline arrangement or remained remodeled. We thus extended the period that we assessed the lymphatic vasculature to 3-4 months following surgical resection. In cross-sectional images of the small intestine (Fig. 5A), we observed the previously documented $40 \%$ increase in villus height by post-operative week 12-15 (Figure 5B; $p<0.0001$ ), serving as a positive control for adaptive remodeling. Lymphatic capillary luminal area in the lamina propria was significantly increased in the distal intestine by $66 \%$ and $70 \%$ in resected mice compared to sham and intraoperative controls, respectively; there was also a pattern of increasing lymphatic area in the proximal intestine (Figure $5 C ; p<0.05, p<0.005$ ). Image analysis for the number lymphatic capillary vessels by both the total length and the total lamina propria area showed no change in the proximal intestine but significantly decreased in the $50 \%$ resected mice compared to both intraoperative and sham controls in the distal intestine (Figure 5D-E).

\section{Mesenteric collecting lymphatic vessel changes after chronic SBR}

In addition to the alterations observed in the distal lymphatic network, we examined the effect of resection on the mesenteric lymphatic vessels. At postoperative week 13 , collecting lymphatic vessels present in the mesentery and emerging from the intestinal wall distal to the anastomosis still showed the 
substantially altered morphology following $50 \%$ SBR versus sham control mice (Figure 6A) that we had earlier observed at day $7(15)$. The resected mice had an average $31 \%$ increase in mesenteric branch width compared to sham controls (Figure 6B; $p<0.05$ ). Furthermore, resected mice had an average $31 \%$ increase in lymphatic budding area into the mesenteric sheath compared to sham controls (Figure $6 \mathrm{C} ; \mathrm{p}<0.05$ ).

\section{Compromise of lymphatic flow after intestinal resection}

The findings above collectively revealed that lymphatic remodeling persisted chronically after SBR. We thus wondered if lymph transport might be altered at these time points. To test the intestinal lymphatic drainage function in SBR, we performed micro injection of a fluorescent marker into Peyer's patches in vivo. Gut that drains to the downstream mesenteric lymphatic network showed compromised flow in resected mice compared to controls (Figure 7A-B; ANOVA $p<0.05$ ). Accumulation of the tracer in the draining lymph node was $78 \%$ and $70 \%$ reduction in mice receiving surgical removal of $50 \%$ of the small bowel compared to nonoperative and sham controls, respectively (Figure 7C; $p<0.005$ ). These data reveal that marked impairment of lymphatic transport accompanies the altered morphology and may account at least in part for the failed absorption of lipid nutrients.

\section{Discussion}

Here, we show by analysis of fecal fat content and by tracing the fate of orally delivered fluorescent cholesterol analogue that dietary lipid absorption is impaired after experimental SBR many weeks beyond the point of surgical resection of the small bowel. Furthermore, we found a significant reduction in the blood cholesterol metabolite campesterol in our mice. This reduction resembles a similar trend in the plasma of SBS patients that have been studied after withdrawal from parenteral nutrition(14). Because campesterol arrives to plasma via absorption from the gut, the finding further supported the conclusion of impaired absorption or transport. Although all nutrient absorption is compromised in SBS patients due to the loss of absorptive surface area, lipid absorption is considered to be the most vulnerable, in part due to compromise of the enterohepatic circulation, decreased bile acid pool, and decreased pancreatic lipase secretion(17-19).

After establishing evidence for failed lipid absorption persisting after SBR in mice, the question became what mechanism is causal. Failed transport of fats may indicate impaired duodenal and jejunal lymph transport, as lipid absorption is most prominent in these sites. Alternatively, impaired lipid transport might point toward reduced capacity of the gut to take up or package fats. The latter appeared unlikely when we found that intestinal remodeling led to elevated expression of genes associated with lipid absorption. The bile acid pool was reduced, and indeed may contribute importantly to reduced lipid uptake. However, we were also compelled to consider the possibility that the lymphatic vasculature was impaired. Besides morphological changes in the lymphatic vasculature after SBR, our studies using fluorescent dextran tracer deposited in the submucosa-draining lymphatic network of the intestine confirmed significantly impaired lymph flow using an approach without confounding possible changes in absorption. 
One week after resection, early lymphatic remodeling in the intestinal mucosal lymphatics is characterized by reduced mucosal lymphatic capillary vessel area(15). Interestingly, after a prolonged period of resection, we have shown that lymphatic capillary area actually increases compared to both intraoperative and sham controls in the distal intestine. We believe that is likely secondary obstruction of flow in the mesentery causing upstream dilation in the intestinal mucosa. We also witnessed this in the mesenteric collecting vessels as the branch width was also dilated after resection. Similar to prior observations seen at POD7, resected mice also had an increase in lymphatic budding area into its mesenteric sheath compared to controls. This budding is normally observed during early development in mice $(20,21)$.

Could failed lymphatic transport account for altered lipid absorption such that lipid remained luminal in the intestine and ultimately appeared in the feces? While the idea that altered lymphatic function might impact absorption across the epithelium may seem counterintuitive, there is strong precedent for it. Preceding studies revealed that loss of lymphatic integrity due to altered signaling in VEGFR3 within lymphatic lacteals leads to increased fecal fat load $(22,23)$. Furthermore, conditions that lead to the closure of intercellular junctions in the lacteals also prevents dietary fat uptake and diverts it to the feces(24).

Overall, we have shown that the early lymphatic remodeling structural changes occur chronically after SBR and contribute to functionally compromised lipid absorption. Further studies are necessary to determine if other lymphatic cargo, such as vitamins and immune cells, are also affected after resection. Perturbations in lymphatic function after SBR likely also contribute to greater delivery of luminal endotoxin into the portal vein, thereby contributing to liver injury(25). Indeed, we have found severe liver injury and fibrosis in resected mice at 15 weeks following SBR when placed on a higher fat diet(26). Liver injury and fibrosis are the most lethal clinical consequences of SBS (27). Manipulations of enteral fat may therefore be a critical therapeutic strategy to prevent this significant morbidity.

These data indicate that intestinal lymphatic vessels significantly remodel in the setting of chronic short bowel syndrome. This remodeling results in impaired intestinal transport of fat via the compromised lymphatic architecture, contributing to decreased fatty acid uptake. We believe that these changes may contribute to the development of IFALD, a major morbidity in patients with SBS.

\section{Materials And Methods}

\section{Animals}

As described previously, C57BL/6J 9-12 week-old male mice were obtained from Jackson Laboratories (Bar Harbor, ME) (15). Prox1-Cre-ER ${ }^{T 2}$ (Jax \# 022075; originally generated by R. Srinivasan and G. Oliver (28)) crossed with Rosa26-tdTomato ${ }^{\text {fl/fl }}$ reporter (Jax \# 007905) 15-20 week male and female mice were orally treated with $20 \mathrm{mg} / \mathrm{ml}$ tamoxifen (Sigma, St. Louis, MO) dissolved in corn oil ( $50 \mu \mathrm{g} / \mathrm{per}$ gram body weight) every other day over two weeks, inducing Cre recombinase activity and downstream tdTomato 
expression. Mice were housed in a temperature controlled, specific pathogen-free unit on a 12-hour lightdark cycle. All mice were fed a liquid diet (PMI Micro-Stabilized Rodent Liquid Diet LD 101; TestDiet, St.

Louis, MO) and water ad libitum. This study was approved by the Washington University in St. Louis Animal Studies Committee (Protocol 20170252 and 20170154) in accordance with the National Institute of Health laboratory animal care and use guidelines. This study is reported in accordance to the ARRIVE guidelines(29).

\section{Operations and harvest}

Mice underwent either a sham control operation, $50 \%$ proximal SBR, or a $75 \%$ proximal SBR, as previously described $(30,31)$. There was also a cohort of mice that did not undergo an operation (non-op), but were treated identically. Mice were placed on liquid diet for 12-24 hours preoperatively to minimize the risk of anastomotic obstruction. In brief, for $50 \%$ and $75 \%$ SBRs, bowel was first exteriorized via a midline laparotomy and then transected 1 to $2 \mathrm{~cm}$ distal to the ligament of Treitz and $12 \mathrm{~cm}$ or $6 \mathrm{~cm}$ proximal to the ileocecal junction, respectively. An end-to-end anastomosis was handsewn with interrupted 9-0 nylon sutures. Sham operations involved a transection with re-anastomosis $12 \mathrm{~cm}$ distal to the ileocecal junction. Intraoperative (IO) resected distal bowel was fixed in $10 \%$ formalin and embedded in paraffin for immunohistochemistry and immunofluorescence. Postoperatively, mice were kept in an incubator and fasted for 12-24 hours before resuming the liquid diet.

\section{RNA extraction and quantitative reverse transcription- polymerase chain reaction}

As described previously, total RNA from proximal and distal intestinal tissue from C57BL/6J mice harvested approximately 1.5 years postoperatively was isolated using RNeasy Mini kits per the manufacturer's protocol (Qiagen, Germantown, MD). Proximal intestine was approximately $6 \mathrm{~cm}$ harvested from $2 \mathrm{~cm}$ past the gastroduodenal junction and distal intestine was the $6 \mathrm{~cm}$ leading up to the ileocecal junction, so as to consistently take the same bowel from all operative types (non-operative, sham, and SBRs)(26). qRT-PCR was conducted using the ABI StepOnePlus Real-Time PCR system with the $A p O B$ primer (forward AAACATGCAGAGCTACTTTGGAG, reverse TTTAGGATCACTTCCTGGTCAAA), FABP2 primer (forward GGTATGGGACAGGCCTTGCT, reverse GGGCATTGTGGTATAGATGACATC), MTTP primer (forward ATGATCCTCTTGGCAGTGCTT, reverse TGAGAGGCCAGTTGTGTGAC), FABP6 primer (forward AGAAGTTCAAGGCTACCGTGAAGA, reverse CCTCCGAAGTCTGGTGATAGTTG), CD36 primer (forward GAGAACTGTTATGGGGCTAT, reverse TTCAACTGGAGAGGCAAAGG), and Abca 1 primer (forward GAAGAGAGCATGTGGAGTTCTT, reverse TACTTTACCAGGCCCAGTTTG; all are from Applied Biosystems, Waltham, MA). The relative mRNA levels were estimated from the equation $2^{-\Delta C t}(\triangle C t=C t$ of target gene minus $C t$ of $18 \mathrm{~S}$ rRNA). Fold changes in the mRNA level of genes were calculated with a control group level set at 1.

\section{Serum free fatty acid, cholesterol, lathesterol, and campesterol levels}


Serum free fatty acid, and cholesterol levels were measured using a commercially available kit (Wako Chemicals, Richmond, VA) on C57BL/6J at 12-15 weeks after SBR. Serum lathesterol and campesterol levels were measured on C57BL/6J mice 12-13 weeks after SBR. The lathosterol and campesterol esters in mouse plasma were hydrolyzed with potassium hydroxide, and total lathosterol and campesterol were extracted with liquid-liquid extraction in the presence of d7-lathosterol and d6-campesterol as internal standards for lathosterol and campesterol, respectively. The plasma samples and 8-point calibration samples were derivatized with nicotinic acid to improve the mass spectrometric sensitivity. The lathosterol and campesterol were separated by reversed phase liquid chromatography and detected with positive multiple-reaction monitoring on an Applied Biosystems Sciex 4000QTRAP tandem mass spectrometer. The peak integration and construction of standard curve were performed with Analyst 1.6.3. The calibration curve was constructed by plotting the peak area ratios of analyte to internal standard versus the corresponding concentrations using $1 / x 2$ weighted least square regression. Serum free fatty acid, and cholesterol levels were performed on mice with confirmed adaptation; adaptation was not assessed in mice tested for serum campesterol and lathosterol levels.

\section{Functional cholesterol transport}

Intestinal uptake of cholesterol was measured using TopFluor-cholesterol (Avanti Polar Lipids, Alabaster, $\mathrm{AL})$ in C57BL/6J mice at post-operative week 31 $(32,33)$. Mice were fasted for six hours overnight and then gavaged with TopFluor ( $8 \mu \mathrm{g} / \mathrm{gm}$ body weight) with methyl- $\beta$-cyclodextrin ( $1 \mathrm{mg} / \mathrm{gm}$ body weight; Sigma-Aldrich, St. Louis, MO). Tail vein serum was measured at time points $0,1,2,4$, and 6 hours after gavage. Methyl- $\beta$-cyclodextrin is given with TopFluor in order to deplete already present plasma membrane cholesterol $(34,35)$. Serum fluorescence was determined using Cytation 5 multiplate reader (Biotek, Winooski, VT). Mice were excluded if fluorescence curve was not achieved secondary to gavage error.

\section{Food consumption and fecal fat content}

Food consumption and fecal fat content were analyzed on mice aged approximately 1 .5years after operation. After acclimation to individual metabolic cages, food intake was measured and feces were collected for two days. As previously described, fecal fat content was determined gravimetrically(36). In brief, dried feces $(0.2 \mathrm{gm})$ was solubilized overnight in $1.6 \mathrm{~mL}$ water and extracted in $5 \mathrm{~mL}$ chloroform:methanol (2:1). The organic phase was transferred into a pre-weighed vial and the homogenate was re-extracted again with $2 \mathrm{~mL}$ of cholorform:methanol (2:1). The organic phase was transferred again and, in total, was dried under nitrogen and reweighed to determine the lipid mass. Percent fat absorption was determined by normalizing the lipid mass to food consumption.

\section{Functional chylomicron transport}

Triglyceride transport was measured in C57BL/6J mice at approximately one-year postoperatively. Mice were fasted for six hours overnight and then gavaged with C16-Bodipy labeled olive oil $(10 \mu \mathrm{g} / \mathrm{gm}$ body weight; Invitrogen, Carlsbad, CA). Tail vein serum was measured at time points 0, 1, 2, 4, and 6 hours after 
gavage. Serum fluorescence was determined using Cytation 5 multiplate reader (Biotek, Winooski, VT). Mice were excluded if fluorescence curve was not achieved secondary to gavage error.

\section{Bile acid absorption}

C57BL/6J mice approximately 1 year after operation were fasted for six hours overnight and then gavaged with $20 \%$ intralipid $(7.5 \mu \mathrm{L} / \mathrm{gm}$ body weight; Sigma-Aldrich; St. Louis, MO). Tail vein serum was measured at time points $0,30,60$, and 120 minutes after gavage. Serum bile acids were measured using a commercially available kit (CrystalChem; Elk Grove Village, IL). Mice were excluded if curve was not achieved secondary to gavage error.

\section{Immunohistochemistry and immunofluorescence}

As described previously, intestinal tissue from 50\% SBR and sham control mice $1 \mathrm{~cm}$ distal to their anastomosis was harvested 12 weeks post-operatively, fixed in $10 \%$ formalin and embedded in paraffin similar to the 10 samples taken from the distal bowel resected in the $50 \%$ SBR mice at operation(15). Additionally, proximal gut was obtained from both sham and 50\% SBR mice 15 weeks after operation at approximately $5 \mathrm{~cm}$ from the gastro-duodenal junction. Longitudinal, $5-\mu \mathrm{m}$ thick sections were created and hematoxylin and eosin stained for villus height measurement to asses for structural adaptation (NIS elements AR 4; Nikon, Melville, NY), which was set at $>15 \%$ increase from IO samples(37). For immunofluorescence, slides were deparaffinized and antigen retrieval (Diva Decloaking solution, Biocare Medical, Concord, CA) was performed under pressure for 10 minutes. Slides were blocked in donkey serum (5\%, Sigma-Aldrich), bovine serum albumin (1\%, Sigma-Aldrich), and Triton-X100 (0.03\%, Pharmacia Biotech). Slides were incubated overnight at $4^{\circ} \mathrm{C}$ with primary antibodies diluted in $0.2 \%$ bovine serum albumin and then secondary antibodies were added for 1 hour. Antibodies used include rabbit anti-mouse Lymphatic vessel endothelial hyaluronic acid receptor (LYVE-1; 1:600, Abcam, ab14917), Cy3-conjugated donkey anti-rabbit IgG (1:400, Jackson ImmunoResearch, 711-165-152) and FITC-conjugated mouse anti-mouse alpha-smooth muscle actin IgG (1:500, Sigma, F3777, Clone \#1A4). A confocal microscope (Leica SPE and SP8) was used to capture images and blinded analysis was performed using Imaris (Bitplane, Switzerland) and FIJI (ImageJ) software (National Institute of Health, Bethesda, MD) of at least $6 \mathrm{~mm}$ of intestine for each sample. LYVE-1 ${ }^{+}$, which is a marker of lymphatic endothelial cells, vessel structures present in the lamina propria were defined as mucosal lymphatics and slides were excluded if staining was of poor quality(38).

\section{Whole mount mesenteric imaging}

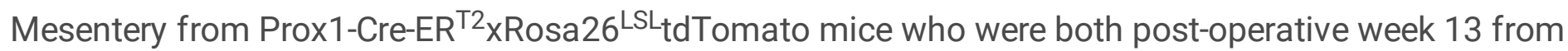
$50 \%$ SBR and sham control operations and also postoperative 1.5 years from $75 \%$ SBR and sham control operations was pinned and fixed in $4 \%$ paraformaldehyde $+30 \%$ sucrose for at least 12 hours and then transferred into phosphate-buffered saline, as described previously(15). The expression of Prospero homeobox protein 1 (PROX1), which governs lymphangiogenesis, is restricted to lymphatic endothelial cells(39). Fluorescent imaging of the lymphatic branches distal to the anastomosis was then performed (Leica M205FA). Blinded image analysis, including average branch width and area of budding structures 
was performed using Imaris (Bitplane, Switzerland) and FIJI (ImageJ) software (National Institute of Health, USA) on the postoperative week $1350 \%$ SBR and sham samples.

\section{Functional Lymphatic Flow}

In mice 12-14 weeks after SBR, anesthetized mice were placed in a custom-built stage to perform intravital recordings using our fluorescence stereoscope, as described previously(40). Physiological conditions were maintained by a temperature-controlled metal stage with 3 temperature sensors (Dual Channel Temperature Controller, Warner Instruments, TC-344C), controlling the temperature independently at 3 sites at $\sim 36-37^{\circ} \mathrm{C}$. Intestine and mesentery were placed over a SYLGARD ${ }^{8} 184$ (Dow Corning, Nidland, MI) stage and securely attached with fine pins (Fine Science Tools, 26002-20). A midline incision of the peritoneum was used to expose the cecum and terminal ileum, along with the mesentery and mesLNs. Mesentery and intestine were kept moist and under controlled temperature with buffer using a peristaltic pump and In-Line Solution Heater (Harvard Apparatus, 64-0102). To address the intestinal lymphatic draining function, we injected $\sim 1.5 \mu \mathrm{L}$ of $2000 \mathrm{kDa}$ FITC-Dextran (Sigma-Aldrich, FD2000S, or Invitrogen, D7137) into a Peyer's patch in the ileum, which was the closest to the anastomosis able to be reached in sham and resected mice and the third from the cecum in non-operative mice. After, serum fluorescence, obtained via tail vein, was determined using Cytation 5 multiplate reader (Biotek, Winooski, VT) at $0,5,10,15,30,45$, and 60 minutes after the Peyer's patch injection and presented as fold change from baseline.

\section{Statistical analysis}

Statistical analysis was performed GraphPad-Prism 6 software (La Jolla, CA). Levels of triglyceride, free fatty acid, cholesterol, campesterol, lathosterol, TopFluor cholesterol, C16-Bodipy, fecal fat, mRNA expression levels, and bile acid levels, were analyzed using a one-way ANOVA with Tukey's multiple comparisons between groups. Intestinal adaptation (increases in villus height) and lymphatic network characteristics were analyzed using the unpaired Student's $t$ test. A p value of $<0.05$ was considered significant. Data are expressed as the mean +/-SEM with significance of multiple comparison analyses displayed.

\section{Abbreviations}

short bowel syndrome (SBS), small bowel resection (SBR), non-operative (non-op), intraoperative (I0), post-operative day (POD)

\section{Declarations}

\section{Acknowledgements}

This work was supported by Pediatric Gastroenterology Training Grant NIH T32 DK077653 (EJO), the NIAID Primary Caregiver Award R37 AI049653 20S1 (Randolph, Onufer), the National Institutes of Health R01 DK119147 (GJR and BWW), the Digestive Diseases Research Core Center of the Washington 
University School of Medicine (NIH \#P30DK52574), and the Children's Surgical Sciences Research Institute of the St. Louis Children's Hospital. This project was supported by the Washington University Institute of Clinical and Translational Sciences which is, in part, supported by the NIH/National Center for Advancing Translational Sciences (NCATS), CTSA grant \#UL1 TR002345. RSC was supported by a fellowship award "FA-2020-01-IBD-1 from the Lawrence C. Pakula, MD IBD Education \& Innovation Fund”.

\section{Conflict of Interest}

The authors have stated explicitly that there are no conflicts of interest in connection with this article.

\section{Author Contributions}

E.J. Onufer, R.S. Czepielewski, G.J. Randolph, and B.W. Warner designed research; E.J. Onufer, R.S. Czepielewski, Y.H. Han, C.M. Courtney, S. Sutton, and A. Sescleifer performed research; E.J. Onufer, R.S. Czepielewski, Y.H. Han, G.J. Randolph, and B.W. Warner analyzed data; E.J. Onufer, G.J. Randolph, and B.W. Warner wrote the paper.

\section{References}

1. Dehmer, J. J., Fuller, M. K., and Helmrath, M. A. (2011) Management of pediatric intestinal failure. Advances in pediatrics 58, 181-194

2. Goulet, O., Ruemmele, F., Lacaille, F., and Colomb, V. (2004) Irreversible intestinal failure. Journal of pediatric gastroenterology and nutrition 38, 250-269

3. Sondheimer, J. M., Cadnapaphornchai, M., Sontag, M., and Zerbe, G. O. (1998) Predicting the duration of dependence on parenteral nutrition after neonatal intestinal resection. The Journal of pediatrics $132,80-84$

4. Tappenden, K. A. (2014) Intestinal adaptation following resection. Journal of Parenteral and Enteral Nutrition 38, 23S-31S

5. Bernier-Latmani, J., and Petrova, T. V. (2017) Intestinal lymphatic vasculature: structure, mechanisms and functions. Nat Rev Gastroenterol Hepatol 14, 510-526

6. Borgström, B. (1960) Studies on intestinal cholesterol absorption in the human. The Journal of clinical investigation 39, 809-815

7. Tilvis, R. S., and Miettinen, T. A. (1986) Serum plant sterols and their relation to cholesterol absorption. The American journal of clinical nutrition 43, 92-97

8. Miettinen, T., Tilvis, R., and Kesäniemi, Y. (1989) Serum cholestanol and plant sterol levels in relation to cholesterol metabolism in middle-aged men. Metabolism 38, 136-140 
9. Miettinen, T. A., Tilvis, R. S., and Kesaniemi, Y. A. (1990) Serum plant sterols and cholesterol precursors reflect cholesterol absorption and synthesis in volunteers of a randomly selected male population. Am J Epidemiol 131, 20-31

10. Nissinen, M. J., Gylling, H., and Miettinen, T. A. (2008) Responses of surrogate markers of cholesterol absorption and synthesis to changes in cholesterol metabolism during various amounts of fat and cholesterol feeding among healthy men. British Journal of Nutrition 99, 370-378

11. Färkkilä, M., and Mieitinen, T. (1988) Plasma lathosterol and campesterol in detection of ileal dysfunction. Scandinavian journal of gastroenterology 23, 19-25

12. Vuoristo, M., Tarpila, S., and Miettinen, T. (1980) Serum lipids and fecal steroids in patients with celiac disease: effects of gluten-free diet and cholestyramine. Gastroenterology 78, 1518-1525

13. Hakala, K., Vuoristo, M., Luukkonen, P., Järvinen, H. J., and Miettinen, T. (1997) Impaired absorption of cholesterol and bile acids in patients with an ileoanal anastomosis. Gut 41, 771-777

14. Pakarinen, M. P., Kurvinen, A., Gylling, H., Miettinen, T. A., Pesonen, M., Kallio, M., Koivusalo, A. I., and Nissinen, M. J. (2010) Cholesterol metabolism in pediatric short bowel syndrome after weaning off parenteral nutrition. Dig Liver Dis 42, 554-559

15. Onufer, E. J., Czepielewski, R., Seiler, K. M., Erlich, E., Courtney, C. M., Bustos, A., Randolph, G. J., and Warner, B. W. (2019) Lymphatic network remodeling after small bowel resection. Journal of pediatric surgery 54, 1239-1244

16. Seiler, K. M., Waye, S. E., Kong, W., Kamimoto, K., Bajinting, A., Goo, W. H., Onufer, E. J., Courtney, C., Guo, J., and Warner, B. W. (2019) Single-cell analysis reveals regional reprogramming during adaptation to massive small bowel resection in mice. Cellular and molecular gastroenterology and hepatology $\mathbf{8}$, 407-426

17. Chandra, R., and Kesavan, A. (2018) Current treatment paradigms in pediatric short bowel syndrome. Clinical journal of gastroenterology 11, 103-112

18. Mouillot, T., Beylot, M., Drai, J., Hillon, P., Gelas, P., Lauverjat, M., Brondel, L., and Chambrier, C. (2020) Effect of bile acid supplementation on endogenous lipid synthesis in patients with short bowel syndrome: A pilot study. Clinical Nutrition 39, 928-934

19. SEIDNER, D., and STEIGER, E. (2005) Managing short bowel syndrome: making the most of what the patient still has. Cleveland Clinic journal of medicine 72, 833

20. Sweet, D. T., Jimenez, J. M., Chang, J., Hess, P. R., Mericko-Ishizuka, P., Fu, J., Xia, L., Davies, P. F., and Kahn, M. L. (2015) Lymph flow regulates collecting lymphatic vessel maturation in vivo. The Journal of clinical investigation 125, 2995-3007 
21. Kume, T. (2015) Lymphatic vessel development: fluid flow and valve-forming cells. The Journal of clinical investigation 125, 2924-2926

22. Nurmi, H., Saharinen, P., Zarkada, G., Zheng, W., Robciuc, M. R., and Alitalo, K. (2015) VEGF-C is required for intestinal lymphatic vessel maintenance and lipid absorption. EMBO Molecular Medicine 7, $1418-1425$

23. Shew, T., Wolins, N. E., and Cifarelli, V. (2018) VEGFR-3 Signaling Regulates Triglyceride Retention and Absorption in the Intestine. Frontiers in Physiology 9

24. Zhang, F., Zarkada, G., Han, J., Li, J., Dubrac, A., Ola, R., Genet, G., Boyé, K., Michon, P., Künzel, S. E., Camporez, J. P., Singh, A. K., Fong, G.-H., Simons, M., Tso, P., Fernández-Hernando, C., Shulman, G. I., Sessa, W. C., and Eichmann, A. (2018) Lacteal junction zippering protects against diet-induced obesity. Science 361, 599-603

25. Han, Y.-H., Onufer, E. J., Huang, L.-H., Sprung, R. W., Davidson, W. S., Czepielewski, R. S., Wohltmann, M., Sorci-Thomas, M. G., Warner, B. W., and Randolph, G. J. (2021) Enterically derived highdensity lipoprotein restrains liver injury through the portal vein. Science $\mathbf{3 7 3}$, eabe6729

26. Onufer, E. J., Han, Y.-H., Czepielewski, R. S., Courtney, C. M., Sutton, S., Randolph, G. J., and Warner, B. W. (2020) Effects of high-fat diet on liver injury after small bowel resection. Journal of pediatric surgery 55, 1099-1106

27. Courtney, C. M., and Warner, B. W. (2017) Pediatric intestinal failure-associated liver disease. Current opinion in pediatrics $29,363-370$

28. Srinivasan, R. S., Dillard, M. E., Lagutin, O. V., Lin, F. J., Tsai, S., Tsai, M. J., Samokhvalov, I. M., and Oliver, G. (2007) Lineage tracing demonstrates the venous origin of the mammalian lymphatic vasculature. Genes \& development 21, 2422-2432

29. Percie du Sert, N., Hurst, V., Ahluwalia, A., Alam, S., Avey, M. T., Baker, M., Browne, W. J., Clark, A., Cuthill, I. C., and Dirnagl, U. (2020) The ARRIVE guidelines 2.0: Updated guidelines for reporting animal research. Journal of Cerebral Blood Flow \& Metabolism 40, 1769-1777

30. Helmrath, M. A., VanderKolk, W. E., Can, G., Erwin, C. R., and Warner, B. W. (1996) Intestinal adaptation following massive small bowel resection in the mouse. Journal of the American College of Surgeons 183, 441-449

31. Wakeman, D., Longshore, S. W., McMellen, M. E., Santos, J. A., Guo, J., Erwin, C. R., and Warner, B. W. (2010) Extent of small bowel resection does not influence the magnitude of intestinal adaptation in the mouse. Journal of Pediatric Surgery 45, 1274-1279

32. Marks, D. L., Bittman, R., and Pagano, R. E. (2008) Use of Bodipy-labeled sphingolipid and cholesterol analogs to examine membrane microdomains in cells. Histochemistry and cell biology 130 , 
33. Hölttä-Vuori, M., Uronen, R.-L., Repakova, J., Salonen, E., Vattulainen, I., Panula, P., Li, Z., Bittman, R., and Ikonen, E. (2008) BODIPY-Cholesterol: A New Tool to Visualize Sterol Trafficking in Living Cells and Organisms. Traffic (Copenhagen, Denmark) 9, 1839-1849

34. Neufeld, E. B., Cooney, A. M., Pitha, J., Dawidowicz, E. A., Dwyer, N. K., Pentchev, P. G., and Blanchette-Mackie, E. J. (1996) Intracellular trafficking of cholesterol monitored with a cyclodextrin. Journal of Biological Chemistry 271, 21604-21613

35. López, C. A., de Vries, A. H., and Marrink, S. J. (2011) Molecular mechanism of cyclodextrin mediated cholesterol extraction. PLoS computational biology 7

36. Aoyama, T., Peters, J. M., Iritani, N., Nakajima, T., Furihata, K., Hashimoto, T., and Gonzalez, F. J. (1998) Altered constitutive expression of fatty acid-metabolizing enzymes in mice lacking the peroxisome proliferator-activated receptor alpha (PPARalpha). The Journal of biological chemistry 273, 5678-5684

37. Wakeman, D., Guo, J., Santos, J. A., Wandu, W. S., Schneider, J. E., McMellen, M. E., Leinicke, J. A., Erwin, C. R., and Warner, B. W. (2012) p38 MAPK regulates Bax activity and apoptosis in enterocytes at baseline and after intestinal resection. American Journal of Physiology-Gastrointestinal and Liver Physiology 302, G997-G1005

38. Jang, J. Y., Koh, Y. J., Lee, S.-H., Lee, J., Kim, K. H., Kim, D., Koh, G. Y., and Yoo, O. J. (2013) Conditional ablation of LYVE-1+ cells unveils defensive roles of lymphatic vessels in intestine and lymph nodes. Blood 122, 2151-2161

39. Wigle, J. T., Harvey, N., Detmar, M., Lagutina, I., Grosveld, G., Gunn, M. D., Jackson, D. G., and Oliver, G. (2002) An essential role for Prox1 in the induction of the lymphatic endothelial cell phenotype. The EMBO journal 21, 1505-1513

40. Czepielewski, R. S., Erlich, E. C., Onufer, E. J., Young, S., Saunders, B., Han, Y.-H., Wohltmann, M., Wang, P. L., Kim, K.-W., and Kumar, S. (2021) Tertiary lymphoid organs drive disrupted gut to lymph node communication through association with collecting lymphatic vessels. Am Assoc Immnol

\section{Figures}



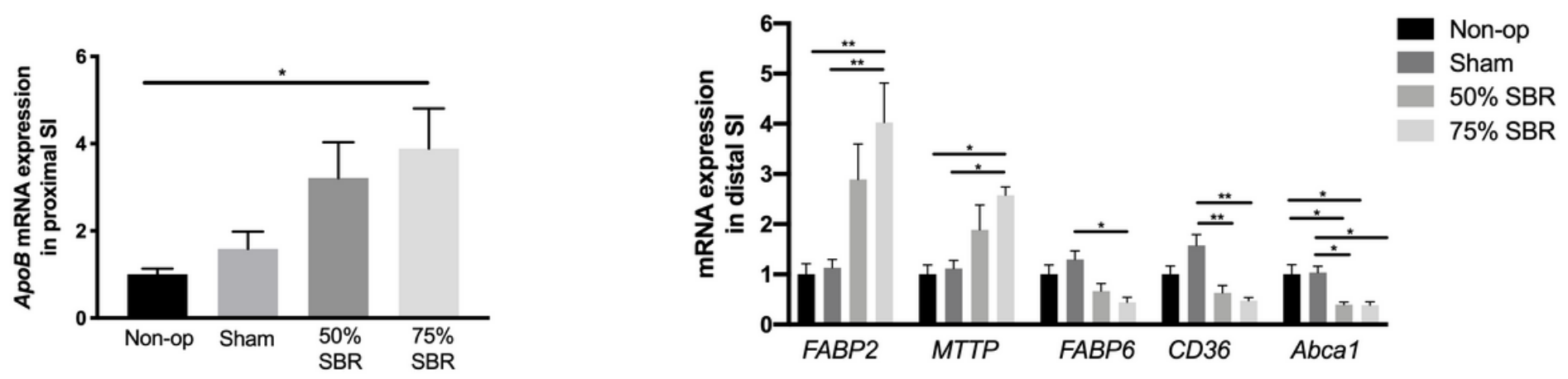

Figure 1

Intestinal fatty acid transporters in the (A) proximal and (B) distal small intestine (SI) in non-op ( $n=5)$, sham ( $n=5), 50 \%$ SBR $(n=5)$, and 75\% SBR $(n=4)$ mice. * $p<0.05,{ }^{*} p<0.005$. 
A

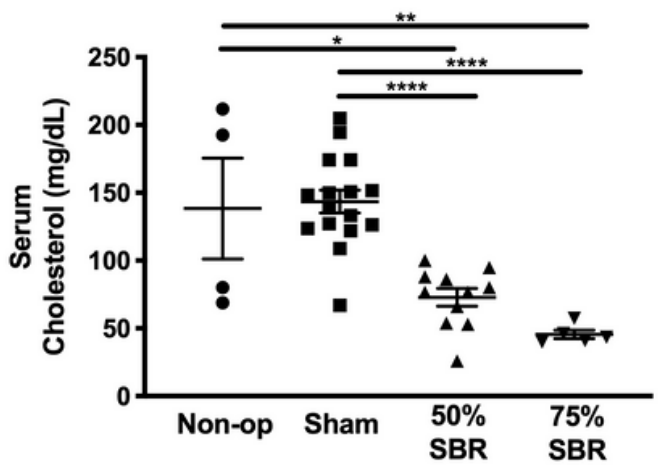

C

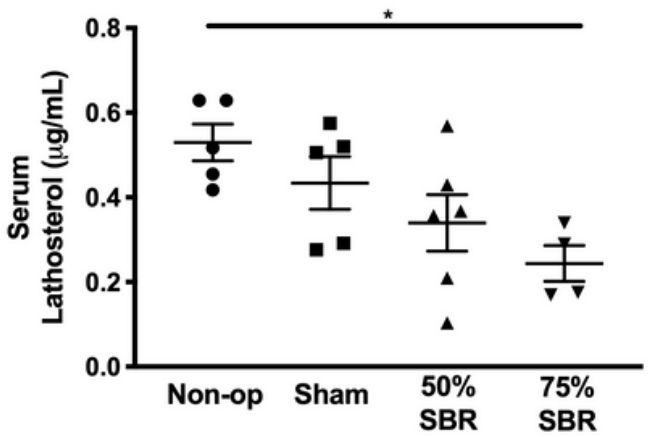

E

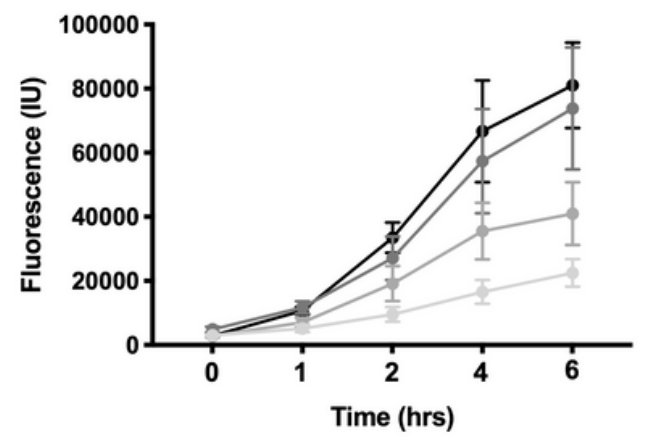

B

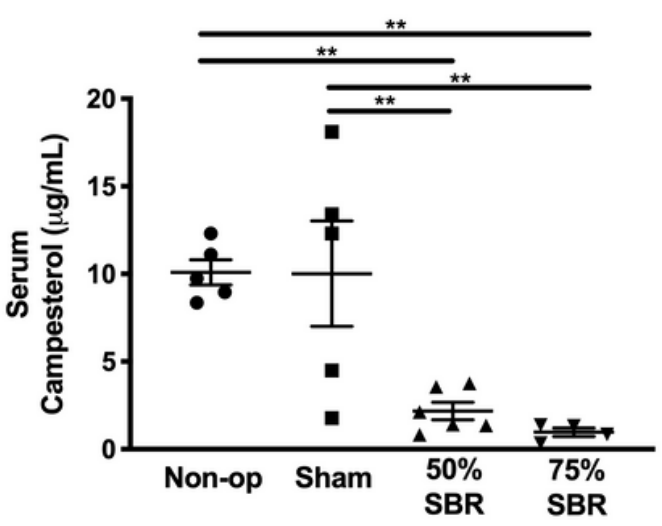

D

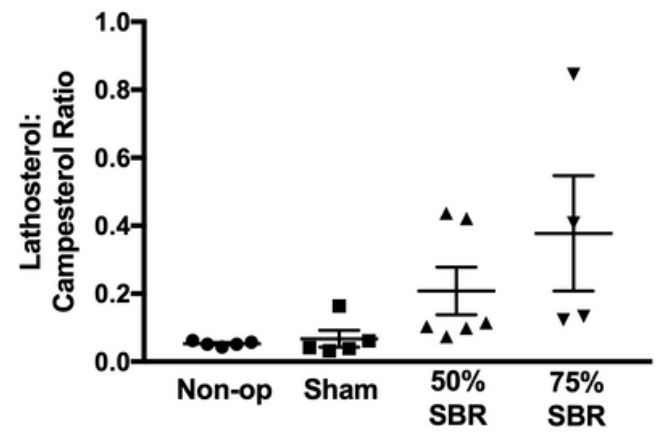

$\mathrm{F}$

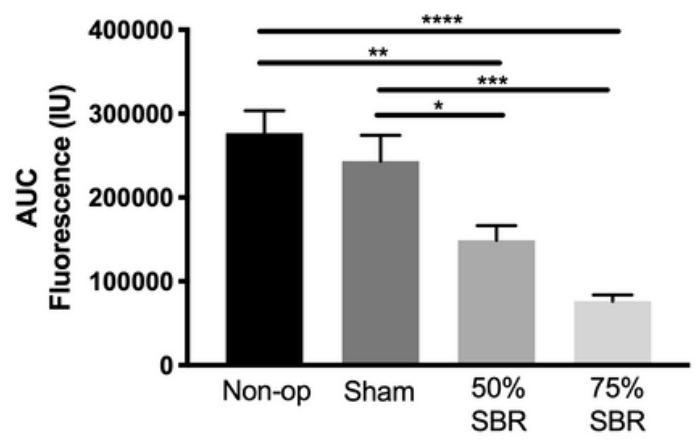

Non-op

Sham

$50 \%$ SBR

$75 \%$ SBR

Figure 2

Effects of SBR on cholesterol and its derivatives. (A) Serum cholesterol levels in non-op $(n=4)$, sham $(n=16), 50 \%$ SBR $(n=11)$, and 75\% SBR $(n=5)$ mice. (B) Serum campesterol levels, (C) lathosterol levels, and (D) lathosterol:campesterol ratios in non-op $(n=5)$, sham $(n=5), 50 \%$ SBR $(n=6), 75 \%$ SBR $(n=4)$ mice. Exogenous absorption of TopFluor-cholesterol assessed via fluorescence over (E) time course and as (F) area under the curve in non-op $(n=5)$, sham $(n=6), 50 \%$ SBR $(n=6)$, and $75 \%$ SBR $(n=5)$ mice. ${ }^{*} p<0.05$, $\star \star x p<0.005,{ }^{* \star} p<0.001, * \star \star \star p<0.0001$. 
A

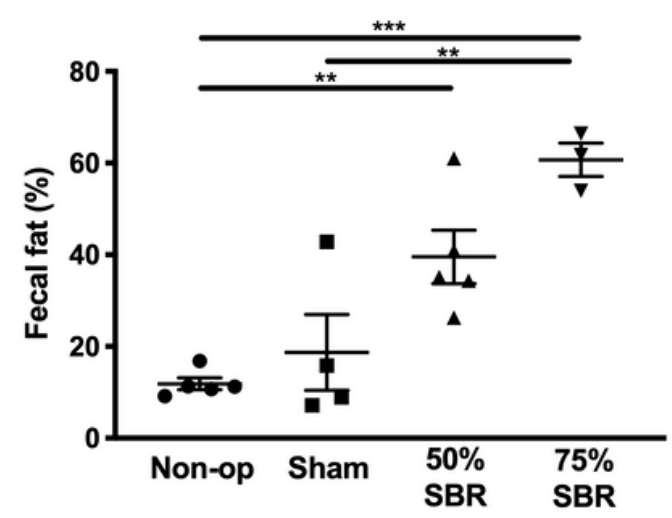

$\mathrm{C}$

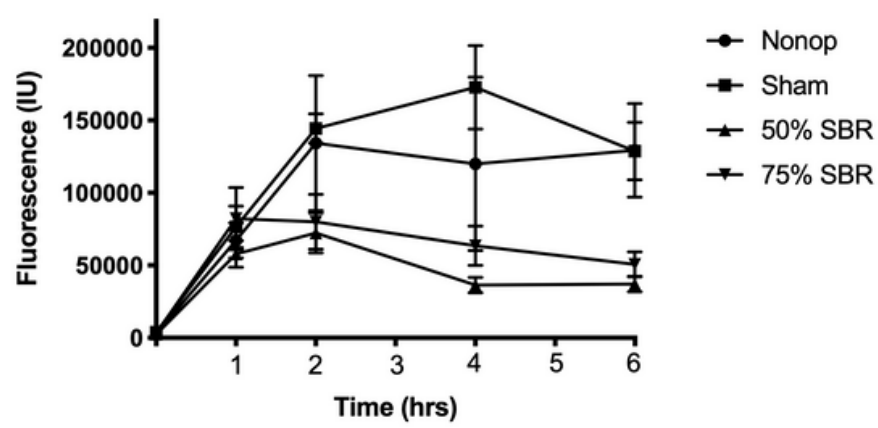

$E$

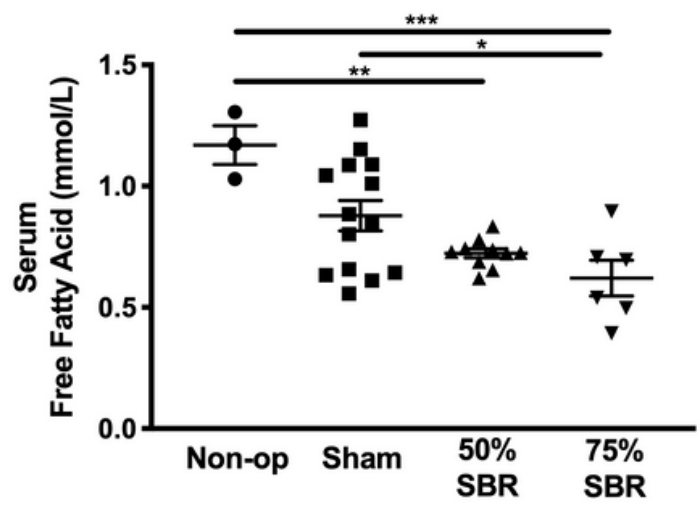

B

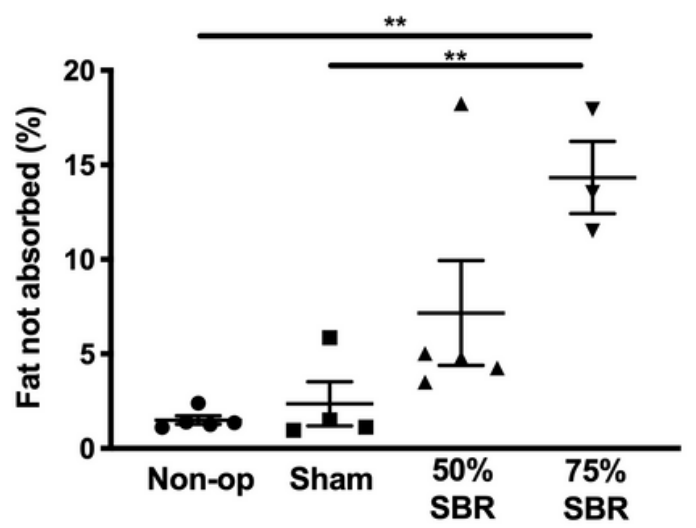

D

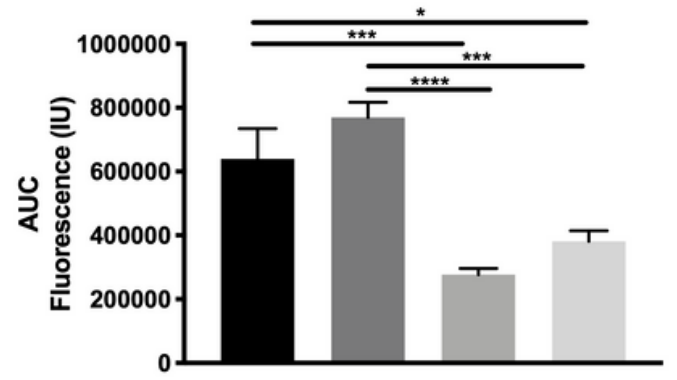

Nonop

Sham

$50 \%$ SBR

$75 \%$ SBR

\section{Figure 3}

(A) Fecal fat and (B) \% fat unabsorbed at approximately 1.5 years after resection in non-op ( $n=5)$, sham $(n=4), 50 \%$ SBR $(n=5)$, and 75\% SBR $(n=3)$ mice. (C) Kinetic C16-Bodipy-labeled olive oil fluorescence and (D) area under the curve in non-op $(n=5)$, sham $(n=7), 50 \%$ SBR $(n=6)$, and 75\% SBR $(n=5)$ mice. $(E)$ Serum free fatty acid levels non-op $(n=3)$, sham $(n=14), 50 \%$ SBR $(n=9)$, and $75 \%$ SBR $(n=6)$ mice. ${ }^{*} \mathrm{p}<0.05, * * \mathrm{p}<0.005,{ }^{* \star *} \mathrm{p}<0.001$. 
A

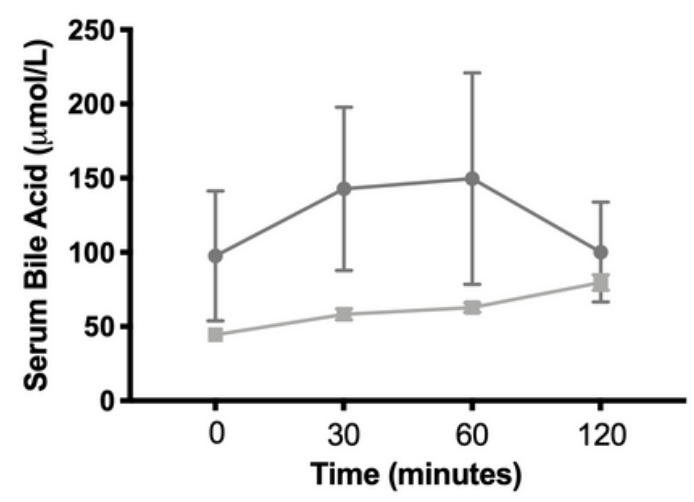

B

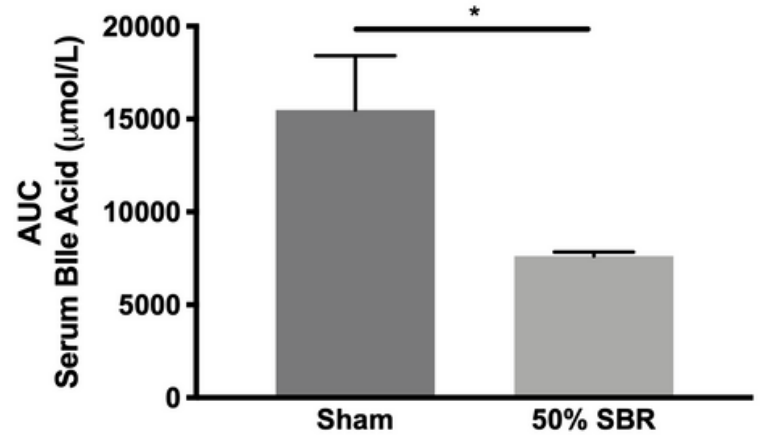

Figure 4

(A) Kinetic bile acid absorption and (B) area under the curve following an oral lipid bolus in sham ( $n=5)$ and $50 \%$ SBR $(n=5)$ mice. * $p<0.05$.

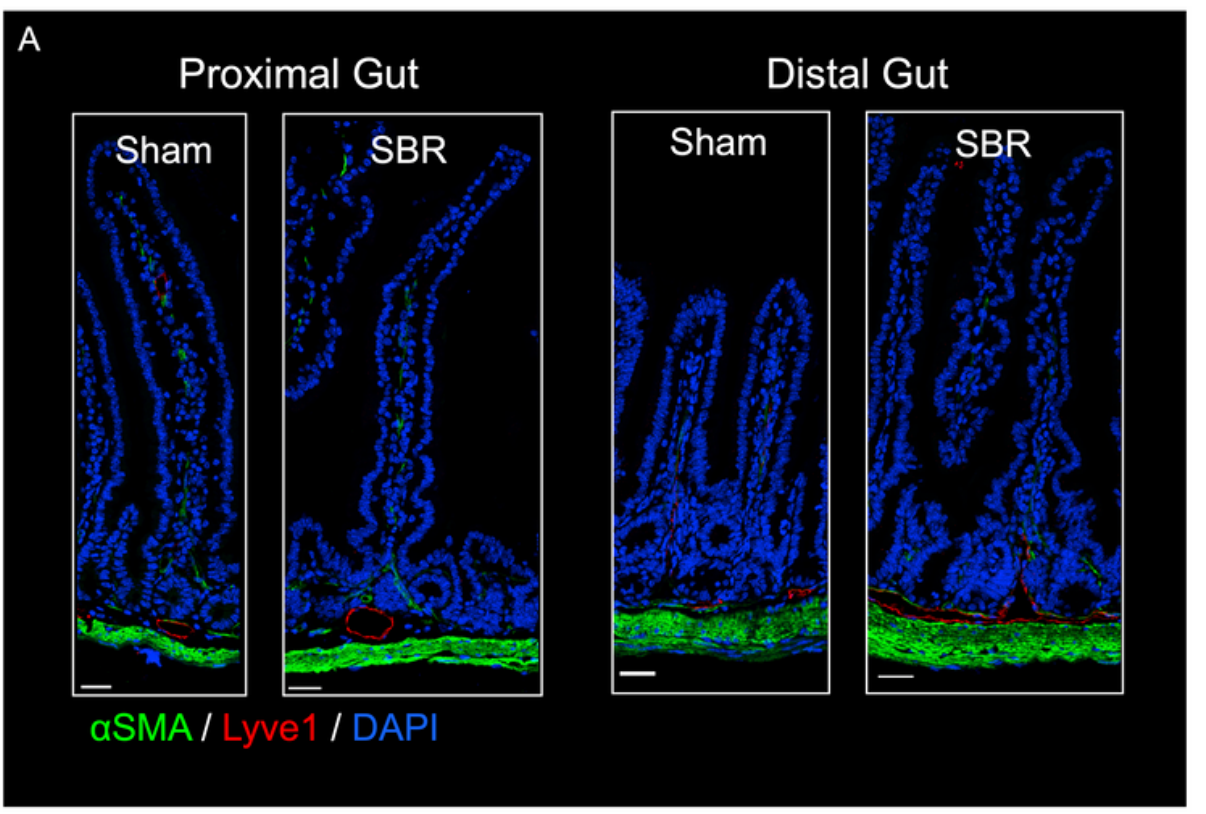

B

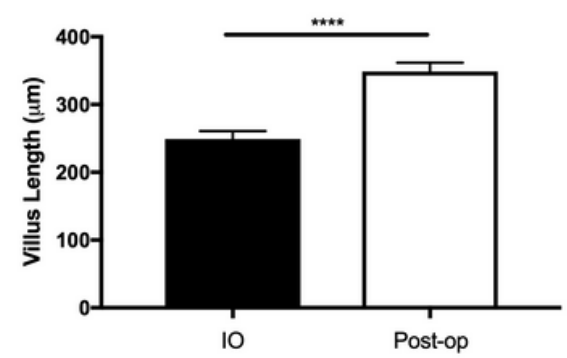

C

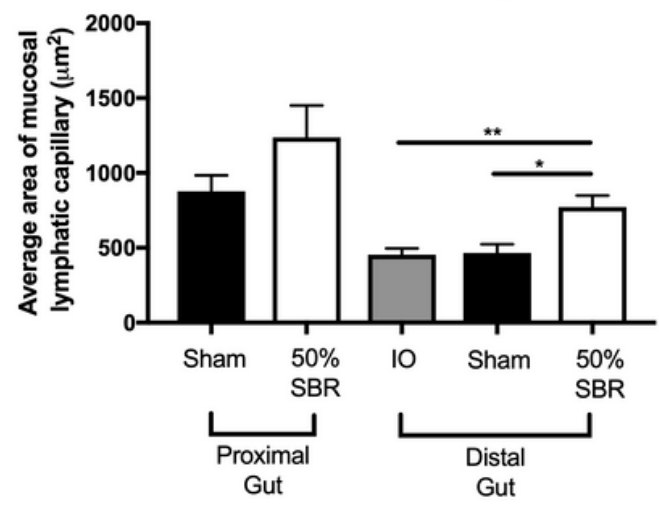

D

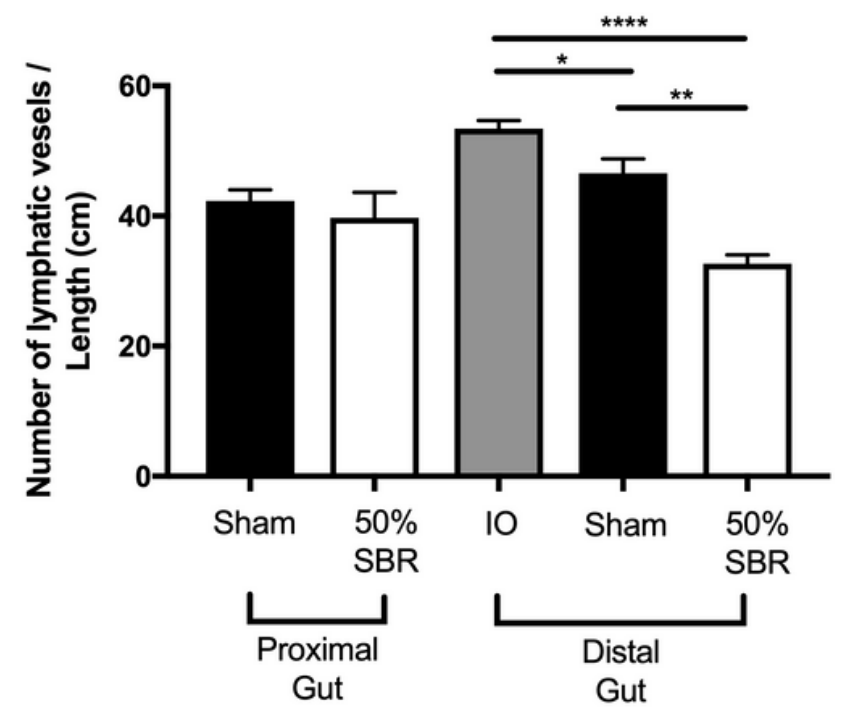

E

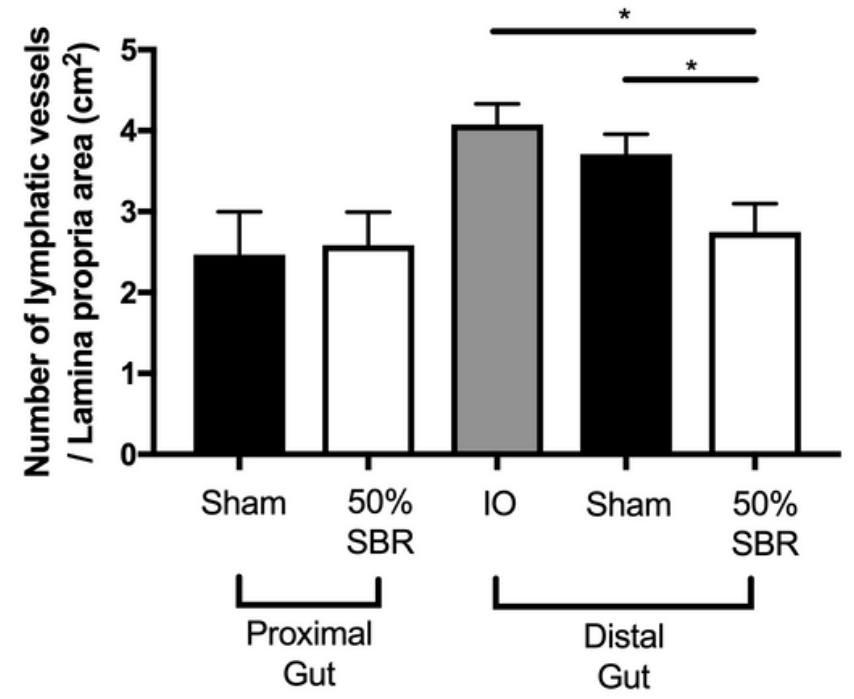




\section{Figure 5}

Chronic mucosal lymphatic capillary changes in SBR vs. sham mice. (A) Representative immunofluorescence images of proximal (left side) and distal (right side) sham and 50\% SBR intestinal tissue sections stained for smooth muscle (alpha-smooth muscle actin, aSMA, green), lymphatics (LYVE1, red), and nuclei (DAPI, blue) on post-operative week 15 and 12, respectively. Lyve1+ vessels were considered mucosal lymphatics when present in the lamina propria (defined as the space between the

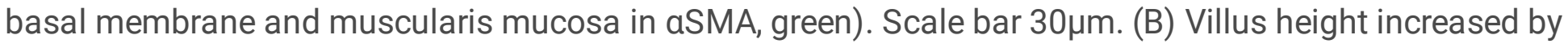
an average of $40 \%$ in $50 \%$ resected mice ( $n=9$ for 10 and Post-op), assuring adaptation. (C) Average area of mucosal lymphatic capillaries in proximal ( $\operatorname{sham~} n=3,50 \%$ SBR $n=3$ ) and distal (intraoperative $n=6$, sham $n=7,50 \%$ SBR $n=4)$ intestine. The number number of mucosal lymphatic capillaries (Lyve1+ vessels as in $A$ ) per (D) length and (E) total area of the lamina propria in proximal and distal intestinal tissue. ${ }^{*} p<0.05, * * p<0.005, * * * * p<0.0001$.
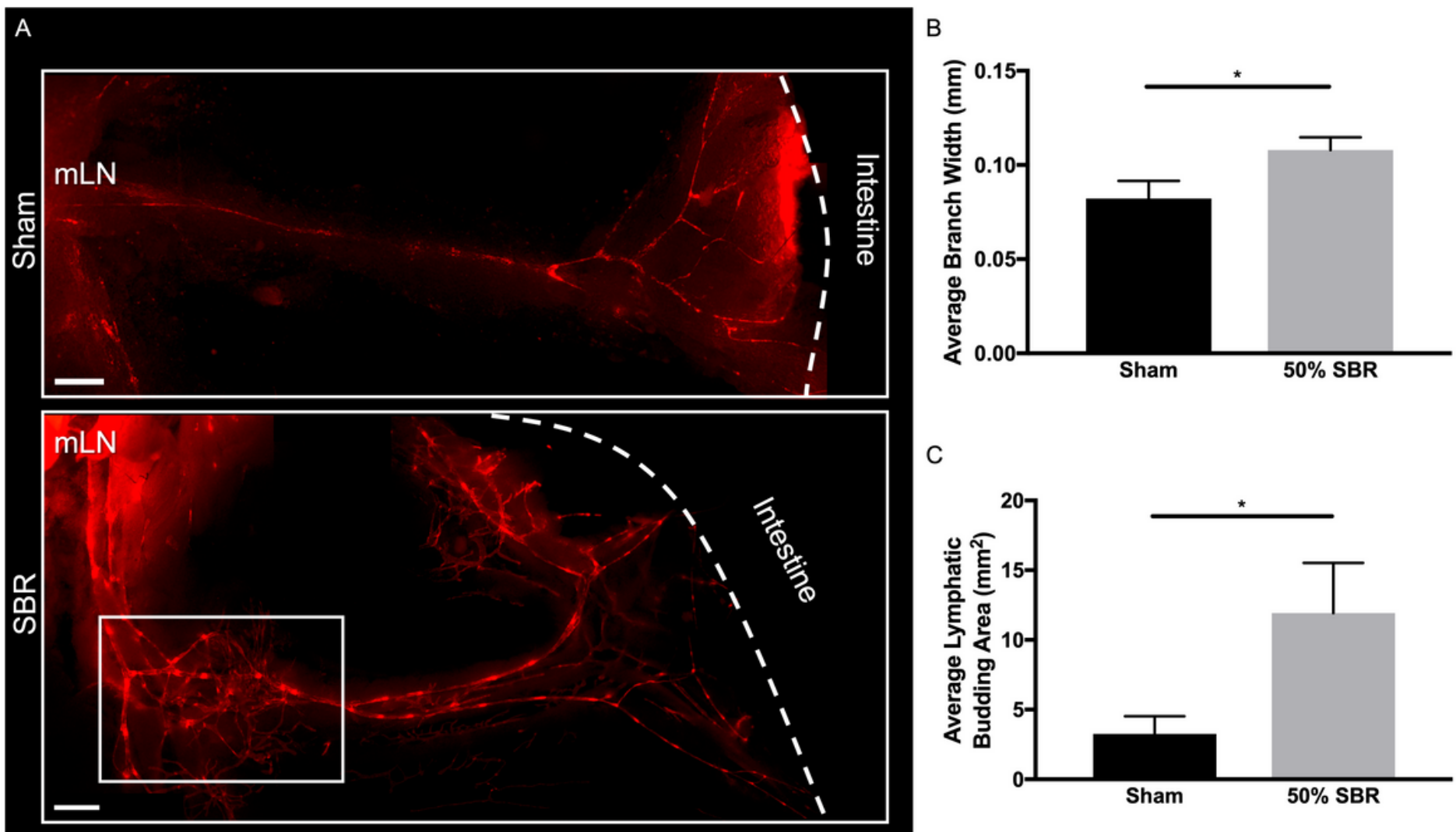

Prox1-CreERT2-tdTomato

\section{Figure 6}

Effect of SBR on mesenteric lymphatic vessels at post-operative week 13. (A) Whole mount fluorescence stereoscope image of collecting lymphatic vessels (Prox1-tdTomato+) (red) in sham and 50\% SBR mice in regions in the ileum-draining mesentery distal to the anastomosis. Example of budding area is outlined by white box; mesenteric lymph node $(\mathrm{mLN})$ is designated to the left and intestine to the right (white

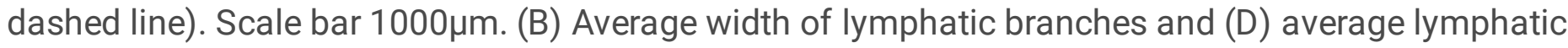


budding area draining the bowel distal to the anastomosis in sham ( $\mathrm{n}=10$ branches in 4 mice) and $50 \%$ $\operatorname{SBR}\left(\mathrm{n}=14\right.$ branches in 4 mice). ${ }^{*} \mathrm{p}<0.05$.

A

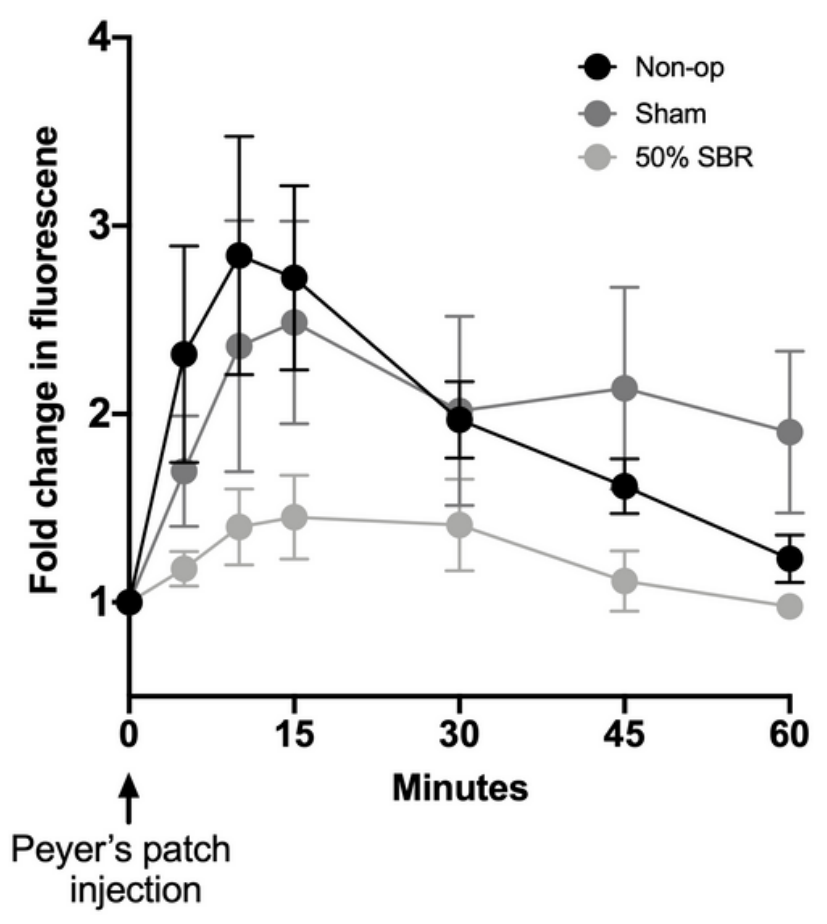

B

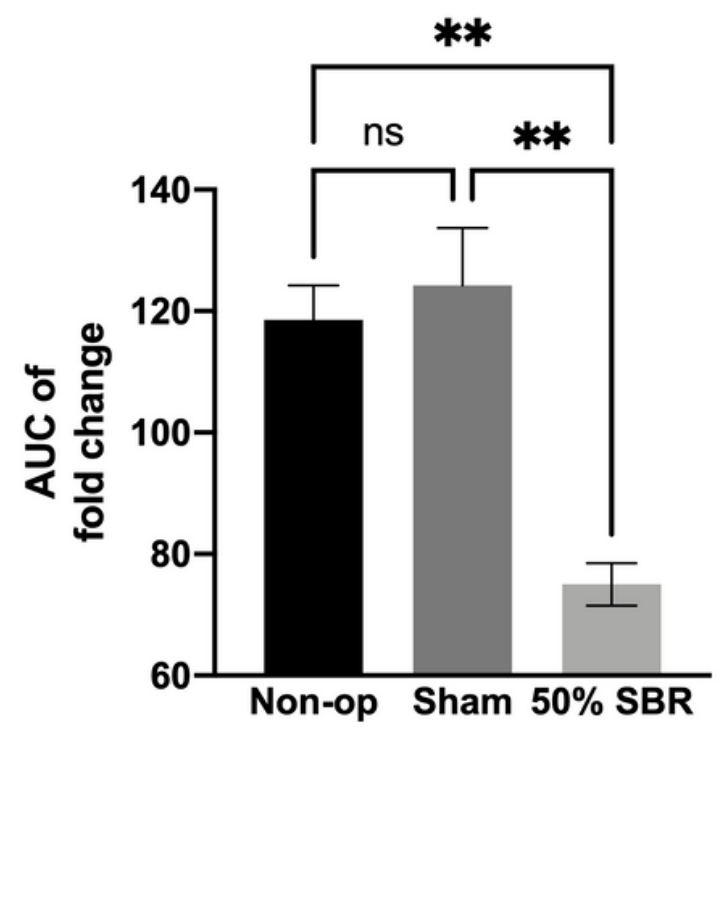

C

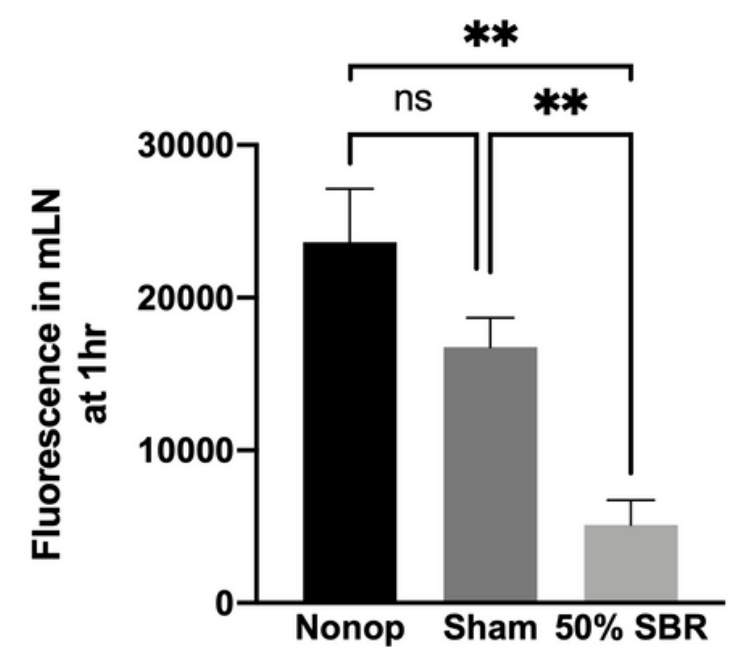

Figure 7

(A) Kinetic measurement of lymphatic flow from Peyer's patch injection to systemic blood by fluorescence, presented in fold change from pre-injection baseline, in non-op $(n=8)$, sham $(n=6)$, and $50 \%$ SBR $(n=4)$ mice as well as the (B) area under the curve. (C) Measurement of fluorescence in the mesenteric lymph node $(\mathrm{mLN})$ at one hour in nonop $(n=3)$, sham $(n=4)$, and $50 \%$ SBR $(n=3)$ mice after Peyer's patch injection. ${ }^{* *} p<0.005$. 